\title{
CONSTRAINED OPTICAL FLOW FOR AERIAL IMAGE CHANGE DETECTION
}

\author{
Nicolas BOURDIS, Denis MARRAUD
}

\author{
EADS France \\ Image Solutions Department \\ Suresnes, France
}

\author{
Hichem SAHBI
}

\author{
CNRS \\ LTCI, Telecom ParisTech \\ Paris, France
}

\begin{abstract}
Nowadays, the amount of video data acquired for observation or surveillance applications is overwhelming. Due to these huge volumes of video data, focusing the attention of operators on "areas of interest" requires change detection algorithms. In the particular task of aerial observation, camera motion and viewpoint differences introduce parallax effects, which may substantially affect the reliability and the efficiency of automatic change detection.

In this paper, we introduce a novel approach for change detection that considers the geometric aspects of camera sensors as well as the statistical properties of changes. Indeed, our method is based on optical flow matching, constrained by the epipolar geometry, and combined with a statistical change decision criterion. The good performance of our method is demonstrated through our new public Aerial Imagery Change Detection (AICD) dataset of labeled aerial images.
\end{abstract}

Index Terms - Change detection, aerial observation, constrained optical flow, epipolar geometry, parallax effects, AICD dataset.

\section{INTRODUCTION}

These last few years have witnessed a rapid development of video acquisition devices, which are becoming cheaper and more efficient. In the context of observation via airborne cameras, the volume of data being acquired is overwhelming human operators and most of it is stored unprocessed or lost. As a consequence, it becomes necessary to develop solutions addressing this bottleneck.

This paper addresses the specific problem of change detection in aerial images, and aims at focusing the operator's attention on areas containing changes. Change detection [1] refers to the problem of detecting significant and possibly subtle changes between a reference and a new (called test) image (e.g. appearing or disappearing buildings or vehicles), while ignoring insignificant ones. General change detection is particularly challenging: difficulties arise when the reference and test images are acquired at distinct dates and with large viewpoint differences. Such conditions usually introduce many environmental changes (illumination, weather, ...)

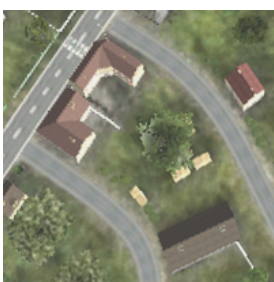

(a)

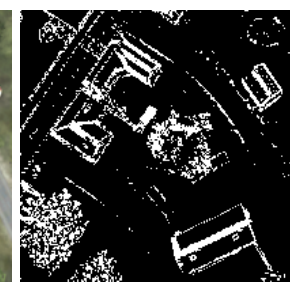

(b)

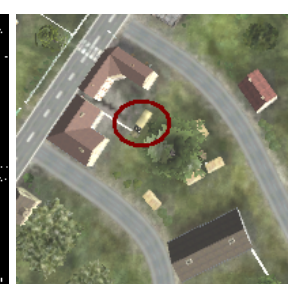

(c)
Fig. 1. This figure shows the reference image (a), the new image (c) with a change near the center (circle) and the image differences (b). True changes and parallax effects are fundamentally indistinguishable using image difference.

and parallax effects due to scene content (trees, buildings, relief) and camera motion. Although a human expert may identify significant changes successfully, such conditions make the task extremely challenging to most of the current automatic approaches.

The method we present focuses on achieving resilience to viewpoint differences and parallax effects while remaining computationally efficient (involving no time-consuming search, for instance [2, 3]) and simple (involving no heavy scene model, like in $[4,5])$ in order to allow for future realtime processing of aerial videos. For that purpose, we introduce a novel approach based on optical flow constrained with the epipolar geometry [6]. More precisely, our contribution is twofold: (i) the underlying mask of change is efficiently estimated using an optical flow algorithm constrained by epipolar geometry, and (ii) our change detection criterion is purely statistical and able to distinguish between real changes and parallax effects.

The remainder of this paper is organized as follows. First, section 2 explains why epipolar geometry provides an appropriate framework in order to cope with parallax effects. Then, section 3 emphasizes on our change detection algorithm, followed in Section 4 by the results obtained on an annotated data-set of aerial images. This benchmark will be publicly available as we believe such data-sets may play a great role in further developments and comparisons of efficient algorithms. 


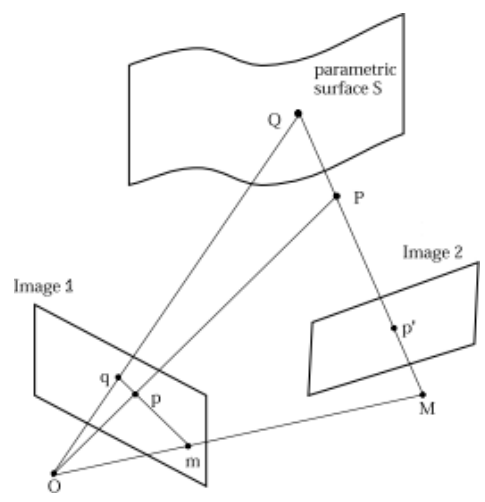

Fig. 2. This figure shows that the residual parallax field after surface alignment is epipolar (reproduced from [7])

\section{PARALLAX EFFECTS AND EPIPOLAR GEOMETRY}

In order to be robust to viewpoint differences, an algorithm should distinguish between real changes and parallax effects. Many existing change detection criteria rely on simple image differences [1], even though this makes true changes and parallax effects indistinguishable, as shown in Figure 1.

As demonstrated in [7], the residual parallax field after surface alignment, or in other words the motion of objects after image registration, is an epipolar field. This is explained by a very clear drawing reproduced in Figure 2 . Let $R_{2}$ be the ray joining a 3D point $P$ and the center of camera 2 (denoted $M) ; R_{2}$ intersects a given arbitrary surface at a $3 \mathrm{D}$ point denoted $Q$. The projection of the ray $R_{2}$ on camera 1 includes the projections of the underlying $3 \mathrm{D}$ points $Q, P$ and $M$ (denoted $q, p$, and $m$ respectively). Since $m$ is the epipole in image 1 , this means that the residual parallax vector $\overrightarrow{q p}$, after registration of both images with respect to an arbitrary surface, is oriented along the epipolar line $q \vec{m}$. Notice that $\overrightarrow{q p}$ can either be directed towards or opposite to the direction of the epipole depending on the relative position of 3D point $P$ and the surface. On the contrary, because true changes appear in only one of the two images, the residual parallax vector will not be defined. Therefore, reasoning on residual parallax vectors, which we obtain using optical flow, provides an effective way to distinguish between true changes and parallax effects.

\section{OVERVIEW OF OUR ALGORITHM}

As discussed in the previous section, images should be aligned with respect to an arbitrary surface. A given test image is registered to the reference one using a homography transformation. First, SURF keypoints are extracted from both images, possible matches are identified using their descriptors and the epipolar geometry is then estimated using the Random Sample Consensus (RANSAC) algorithm. No-

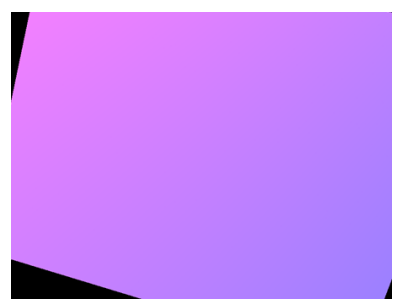

(a)

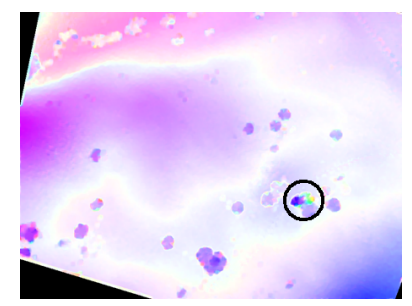

(b)
Fig. 3. This figure presents the color orientation images corresponding to the true epipolar field (a) and the raw optical flow results (b). Hue encodes vector angles while saturation codes for vector norms, value being constant and equal to 255 . We can see that angles (hues) are very similar in both images, except for the change (circle) and a few little areas, even without constraining the optical flow to follow epipolar lines. Enforcing the epipolar geometry constraint removes most of the remaining false alarms.

tice that this step may be accelerated if camera parameters are roughly known both for the reference and the test images (which is usually the case in the context of our applications). Afterwards, more accurate keypoint matches, based on the epipolar geometry, together with RANSAC are again used in order to estimate the homography transformation.

In order to estimate residual parallax vectors, we use the optical flow algorithm described in [8], which is fast and provides a dense vector field. Experiments showed that although most of the optical flow vectors are consistent with the epipolar field (see Figure 3), some of them are spurious and are a source of false alarms in the resulting change mask. Consequently, we constrain, in our algorithm, optical flow vectors to be collinear to the epipole direction. Then we detect changes using a likelihood ratio test on the local matching score.

Let $X$ be a random variable standing for a pixel into a test image. Let $H_{0}$ denote the "null hypothesis" meaning that $X$ is unchanging (according to a well established ground truth); the alternative hypothesis (denoted $H_{1}$ ) stands for the converse (i.e., $X$ is changing). Now, we consider the following likelihood ratio and its corresponding threshold:

$$
\begin{gathered}
L(X)=\frac{P\left(\epsilon(X, \psi(X)) \mid H_{1}\right)}{P\left(\epsilon(X, \psi(X)) \mid H_{0}\right)} \\
\tau=\frac{P\left(H_{0}\right)\left(C_{10}-C_{00}\right)}{P\left(H_{1}\right)\left(C_{01}-C_{11}\right)}
\end{gathered}
$$

where $\epsilon$ denotes an error function and $\psi$ is the optical flow mapping which given $X$ in the test image, finds its unique matching point in the reference image. $C_{i j}$ is a cost associated with making a decision in favor of hypothesis $H_{i}$ when $H_{j}$ is true. The decision as whether $X$ is changing depends on the sign of $L(X)-\tau$. In order to model $P\left(\epsilon(X, \psi(X)) \mid H_{1}\right)$ 


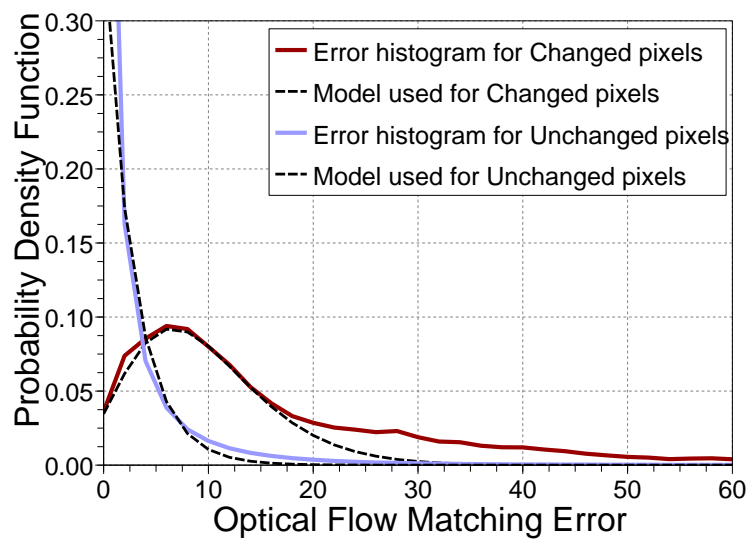

Fig. 4. This figure describes the probability density functions of optical flow matching errors for Changed and Unchanged pixels.

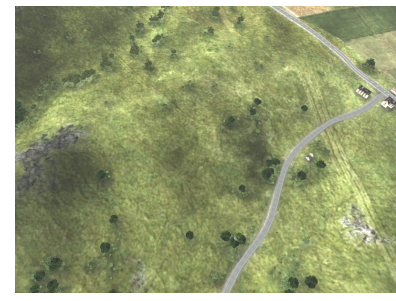

(a)

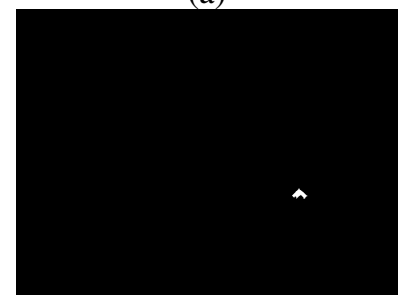

(c)

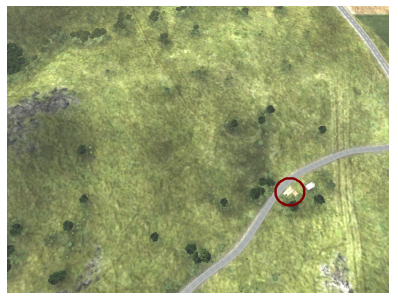

(b)

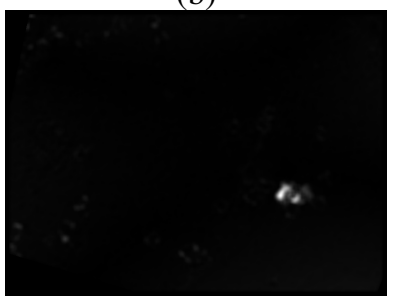

(d)
Fig. 5. This figure shows the reference image (a), test image (b) with change (circle), ground truth (c) and estimated (d) change masks.

and $P\left(\epsilon(X, \psi(X)) \mid H_{0}\right)$, we analyze the optical flow matching error $\epsilon(X, \psi(X))$ through a population of changing and unchanging points using our dataset (see section 4$)$. The distributions of these errors are described in Figure 4 with the corresponding models.

Figure 5 illustrates an example of change detection results obtained on a test image. As we can see, the optical flow matching score corresponds very accurately to the change mask of the ground truth. In mono-thread mode, this result was estimated in less than 3 seconds on a standard $2.4 \mathrm{GHz}$ PC.

\section{EVALUATION}

In order to evaluate our approach quantitatively, we built the Aerial Imagery Change Detection (AICD) dataset using re-

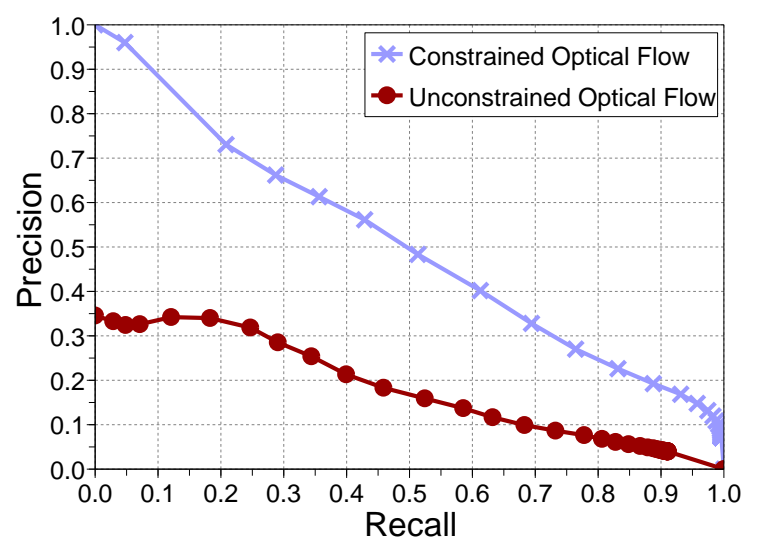

Fig. 6. This figure presents Precision/Recall curves for our method with and without epipolar geometry constraint. The performance is reported on our new AICD dataset.

alistic aerial images rendered artificially ${ }^{1}$. A hundred scenes were created, with and without changes and illumination differences. Although reference and test images include illumination variations, dealing with hard shadows is out of the scope of this paper. Every scene was rendered from five different viewpoints, the last four viewpoints being more and more distant from the first one (by steps of 10 degrees), enabling the analysis of the influence of viewpoint difference on change detection performances. In addition, the true pixelbased change mask is available for each viewpoint, allowing an objective comparison of performances. This dataset will be made publicly available ${ }^{2}$ to enable benchmarking of change detection algorithms.

In order to compare detection results with respect to the ground truth, we define a precision recall criterion at the component level (instead of the pixel level). This criterion is based on intersecting connected components which is more relevant to our objective of attention focusing than a pixel-based comparison.

Figure 6 compares these precision recall performances obtained for a viewpoint difference of 10 degrees, using the method presented above with and without the epipolar geometry constraint. These curves show that constraining the optical flow algorithm using the epipolar geometry makes it possible to achieve far better performance than leaving the optical flow unconstrained, especially the number of false alarms. Indeed, unconstrained optical flow may result into wrong matches between reference and test images. Constraining the optical flow using the epipolar geometry reduces such errors and thereby the false alarm rate.

Notice that the curve obtained without the epipolar geometry constraint converges to the bottom-left corner of the diagram in Figure 6. This behavior corresponds to a scoring

\footnotetext{
${ }^{1}$ We used the built-in rendering engine of the serious game Virtual Battle Station 2, edited by BISimulations.

${ }^{2}$ The dataset will be hosted on ComputerVisionOnline.com.
} 


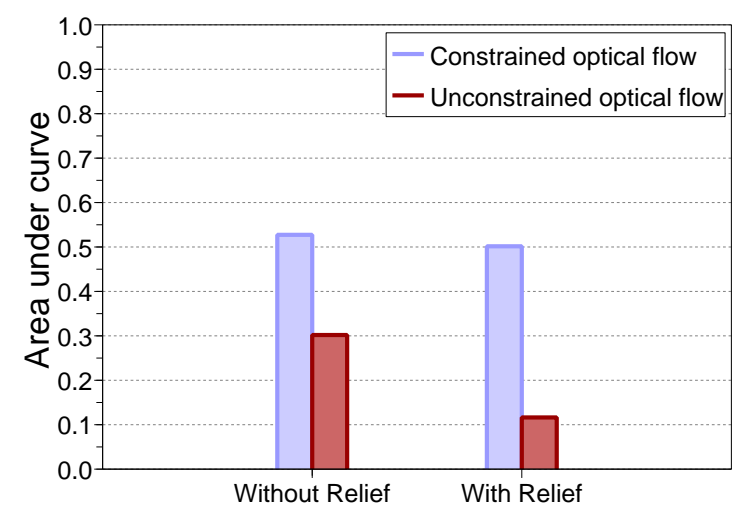

Fig. 7. This figure compares the area under Precision vs Recall curves for the method with and without epipolar geometry constraint, on scenes with or without ground relief.

function whose highest scores occur on false alarms instead of desired detections. This usually means that the considered scoring function cannot appropriately handle some of the provided examples.

As shown in Figure 6, considering the epipolar geometry constraint attenuates these effects, as it aligns the orientation of optical flow to match the true direction, which makes the results more reliable. Figure 7 shows performances obtained on two subsets of images including scenes with and without relief. The underlying ground truth (relief vs. non relief) was generated using a hard decision on the rate of outliers during the estimation of homography with RANSAC (see section 3); resulting into two subsets of comparable sizes. Figure 7 shows that the performance does not significantly decrease in the constrained case, whereas in the unconstrained one, the performance drops by $50 \%$. These results demonstrate that the proposed approach increases robustness to the presence of relief in images, and more generally to poor preliminary registrations between images.

Finally, using the five viewpoints available for each scene, we analyze the performances of our approach with respect to viewpoint variation. Figure 8 shows a consistent gain of the constrained approach compared to the unconstrained one. Limited variations of the viewpoint angle (10 degrees in practice) degrade the performance by less than $20 \%$ for the constrained case while for the unconstrained one, the performance decreases by more than $50 \%$. For viewpoint angles larger than 10 degrees, the two methods show similar behaviors. This may result from the presence of challenging occlusions, due to important viewpoint variations, thereby introducing many false detections.

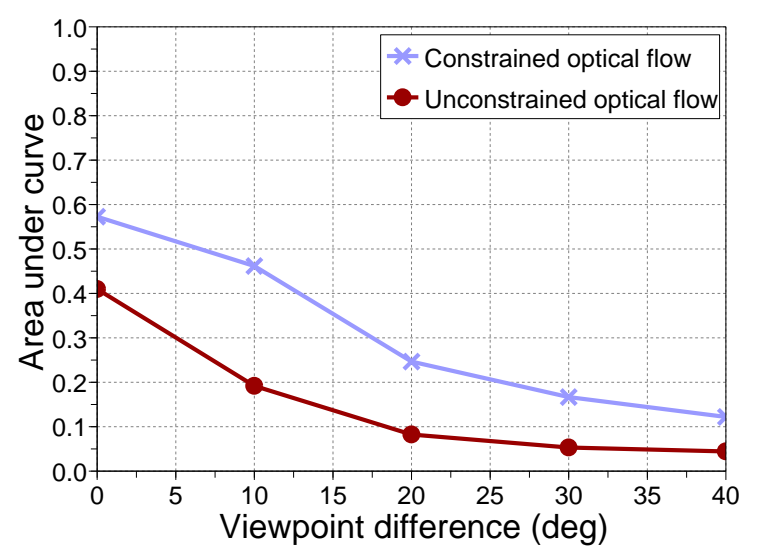

Fig. 8. This figure compares the area under Precision/Recall curves of our method with and without epipolar geometry constraint and for various viewpoint angles.

\section{CONCLUSION}

In this paper, we introduced a change detection algorithm which successfully handles challenging aerial images with strong parallax effects, reliefs and illumination variations while being simple and fast. Obtained results are satisfactory both in terms of visual inspection and Precision/Recall measures. Indeed, our method demonstrates its ability to achieve good robustness to relief and viewpoint changes.

It is known that strong shadows and occluded objects are also challenging for change detection. As a future work, we will address these issues for the purpose of achieving more effective and real-time change detection in aerial videos.

\section{REFERENCES}

[1] R.J. Radke, S. Andra, O. Al-Kofahi, and B. Roysam, "Image change detection algorithms: a systematic survey," IEEE Transactions on Image Processing, vol. 14, no. 3, pp. 294, 2005.

[2] S. Watanabe and K. Miyajima, "Detecting building changes using epipolar constraint from aerial images taken at different positions," in Proceedings of the International Conference on Image Processing (2001), 2001, vol. 2, pp. 201-204.

[3] M. J. Carlotto, "Detecting change in images with parallax," in Society of Photo-Optical Instrumentation Engineers (SPIE) Conference Series, 2007, vol. 6567, p. 42.

[4] T. Pollard and J.L. Mundy, "Change detection in a 3-d world," in Proceedings of the IEEE Conference on Computer Vision and Pattern Recognition, 2007, p. 1.

[5] A. Buchanan, "Novel view synthesis for change detection," in 6th EMRS DTC Conference, 2009.

[6] R. Hartley and A. Zisserman, Multiple view geometry in computer vision, Cambridge University Press, 2nd edition, 2003.

[7] R. Kumar, P. Anandan, and K. Hanna, "Shape recovery from multiple views: A parallax based approach," Proceedings of the International Conference on Pattern Recognition, 1994.

[8] G. Farneback, "Two-frame motion estimation based on polynomial expansion," Image Analysis, p. 363, 2003. 